Valeria Calcaterra*, Rachele De Giuseppe, Ginevra Biino, Melissa Mantelli, Sonia Marchini, Giulia Bendotti, Alexandra Madè, Maria Antonietta Avanzini, Chiara Montalbano, Gianguido Cossellu, Daniela Larizza and Hellas Cena

\title{
Relation between circulating oxidized-LDL and metabolic syndrome in children with obesity: the role of hypertriglyceridemic waist phenotype
}

https://doi.org/10.1515/jpem-2017-0239

Received June 18, 2017; accepted October 4, 2017; previously published online November 11, 2017

\section{Abstract}

Background: The association between oxidative stress (OS) and metabolic syndrome (MetS) has been reported in adults. We analyzed the relation between circulating oxidized lowdensity lipoproteins (Ox-LDL) and MetS in pediatric ages in order to define whether plasma Ox-LDL levels are correlated to obesity and whether oxidative damage, using serum Ox-LDL levels as a proxy, are associated with MetS.

Methods: We enrolled 178 children (11.8 \pm 2.6 years). On the basis of a body mass index (BMI) threshold, the subjects were classified as: normal weight BMI $<75$ th percentile; overweight BMI 75-97th percentile; obese BMI $>97$ th percentile. Patients were classified as having MetS if they met three or more of the following criteria for age and sex: BMI $>97$ th percentile, triglyceride levels $>95$ th percentile,

*Corresponding author: Dr. Valeria Calcaterra, Department of Maternal and Children's Health, Pediatric Endocrinology Unit, Fondazione IRCCS Policlinico S. Matteo and University of Pavia, Department of Internal Medicine, P.le Golgi n.2, 27100 Pavia, Italy, Phone: +390382502930, Fax: +390382527976,

E-mail:v.calcaterra@smatteo.pv.it

Rachele De Giuseppe, Sonia Marchini, Giulia Bendotti and Hellas Cena: Unit of Human Nutrition and Dietetics, Department of Public Health, Experimental and Forensic Medicine, University of Pavia, Pavia, Italy

Ginevra Biino: Institute of Molecular Genetics, National Research Council of Italy, Pavia, Italy

Melissa Mantelli and Maria Antonietta Avanzini: Immunology and Transplantation Laboratory/Cell Factory/Pediatric Hematology/ Oncology Department, Fondazione IRCCS Policlinico S. Matteo, Pavia, Italy

Alexandra Madè, Chiara Montalbano and Daniela Larizza: Pediatric Endocrinology Unit, Department of Internal Medicine, University of Pavia and Department of Maternal and Children's Health, Fondazione IRCCS Policlinico San Matteo, Pavia, Italy Gianguido Cossellu: Department of Biomedical, Surgical and Dental Sciences, University of Milan, Fondazione IRCCS Ca' Granda, Ospedale Maggiore Policlinico, Milano, Italy high-density lipoprotein (HDL) cholesterol level $<5$ th percentile, systolic blood pressure (SBP) and/or diastolic blood pressure (DBP) $>95$ th percentile and impaired glucose tolerance.

Results: Obese children showed increased MetS prevalence $(p=0.001)$ and higher Ox-LDL levels compared to normal- and overweight subjects $(\mathrm{p}<0.05)$, with a limited relation between Ox-LDL and MetS $(p=0.06)$. Waist-to-height ratio $(\mathrm{W} / \mathrm{HtR})(\mathrm{p}=0.02)$, triglycerides $(\mathrm{TG})$ $(p=0.001)$ and LDL-cholesterol $(p<0.001)$ resulted independent predictors of increased plasma Ox-LDL levels.

Conclusions: Oxidative damage was correlated with a hypertriglyceridemic waist phenotype and can be a precocious marker of MetS and cardiometabolic risk in obese children.

Keywords: children; metabolic syndrome (MetS); obesity; oxidative damage; oxidized-low-density lipoproteins (Ox-LDL).

\section{Introduction}

Metabolic syndrome (MetS) is a common and complex disorder combining obesity, dyslipidemia, hypertension and insulin resistance. Once considered typical of adulthood, MetS is also currently a serious, social and health-related problem in pediatric ages [1,2].

Childhood obesity is an independent predictor for the onset of MetS, and its prevalence increases with the degree of obesity [3, 4]. A child with obesity is at higher risk of developing MetS and, consequently, is at a higher risk of cardiovascular incidents later in life, that can only partially be explained by its components [5-7].

There is evidence that low-grade inflammation and high oxidative stress (OS) are associated with this risk. The increased oxidative environment contributes to a chronic inflammatory condition in the body and builds primary risk factors for the development of several diseases such as diabetes and has cardiovascular complications $[7,8]$. On 
the other hand, the increased OS in obesity and metabolic disorders causes progression and sustenance of inflammatory conditions upregulating redox signaling pathways and altering gene expression of inflammatory markers, kicking off a vicious circle [9-12].

Oxidized low-density lipoproten(Ox-LDL) cholesterol is a LDL oxidative modification product. Ox-LDL is an additional cardiovascular risk factor and plays an important role in atheromatous plaque formation [8]; its levels correlate with the increasing severity of a disease (e.g. stable angina vs. unstable angina vs. myocardial infarction). Moreover, Ox-LDL is also considered a reliable marker of oxidative damage to lipids, by the European Food Safety Authority Panel on Dietetic Products, Nutrition and Allergies, when measured with appropriate specificity immunoassays [13].

Recent data suggest that increased OS in adipose tissue is an early instigator of MetS and that the redox state in adipose tissue is potentially a useful therapeutic target for the obesity-associated MetS [11, 12]. Data on the association between Ox-LDL levels and MetS have been reported in adults [11, 12]; however, in pediatric ages data are very limited [14].

We analyzed the relation between circulating Ox-LDL and MetS in pediatric ages in order to define whether plasma Ox-LDL levels are correlated to obesity and whether oxidative damage, using serum Ox-LDL levels as a proxy, are associated with MetS.

\section{Patients and methods}

We enrolled 178 Caucasian children and adolescents aged $11.8 \pm 2.6$ years ( 88 females and 90 males).

On the basis of a body mass index (BMI, calculated as body weight in kilograms divided by body height in meters squared) threshold, according to age-sex percentiles [15], subjects were classified as: normal weight BMI $<75$ th percentile (according to ISPED classification); overweight BMI 75-97th percentile; obese BMI $>97$ th percentile (according to the World Heath Organization [WHO]). This classification was preferred because of the highest sensitivity in identifying obese subjects with clustered cardiometabolic risk factors [16].

Subjects were referred to our institution for auxological evaluation or obesity by their general practitioner or by their primary care pediatric consultant, between November 2015 and December 2016. Exclusion criteria were any known secondary syndromes with or without obesity, any ongoing medical therapy and concomitant chronic or acute illnesses.

\section{Methods}

Auxological parameters evaluation: Physical examination of the patients included weight, height, waist circumference (WC), pubertal stage according to Marshall and Tanner [17, 18] and blood pressure measurement. BMI and waist-to-height ratio (W/HtR) were also calculated.

Weight was measured with participants not wearing shoes and in light clothing, standing upright in the center of the scale platform facing the recorder, hands at sides and looking straight ahead.

Standing height was measured using a Harpenden stadiometer with a fixed vertical backboard and an adjustable head piece. The measurement was taken on the child in an upright position, without shoes, with their heels together and toes apart, hands at sides, aligning the head in the Frankfort horizontal plane. The child was instructed to stand as tall as possible, taking a deep breath, and holding this position to capture the result.

Waist circumference was measured to the nearest centimeter with a flexible steel tape measure with participants standing, with crossed arms, placing the hands on opposite shoulders. After gently exhaling, the abdominal waist circumference was measured on the horizontal plane between the lowest portion of the rib cage and the uppermost lateral border of the right ilium. Visceral adiposity was defined for W/HtR $>0.5$ [19].

Systolic blood pressure (SBP) and diastolic blood pressure (DBP) blood pressures were measured twice using a mercury sphygmomanometer, after the participant sat comfortably for $5 \mathrm{~min}$, with an appropriately sized cuff on the right arm, which was slightly flexed at heart level. The second BP measurement was used for analysis. Elevated SBP or DBP values were defined when exceeding the 95th percentile for age and sex [20].

Biochemical parameters evaluation: In all patients, blood measures included fasting blood levels of glucose, insulin, total cholesterol, HDL, cholesterol, triglycerides (TG), Ox-LDL.

Insulin resistance was calculated by homeostasis model assessment for insulin resistance (HOMA-IR) [21].

Serum glucose was measured using the hexokinase-G-6-PDH method (Abbott Diagnostics, Rome, Italy). Total cholesterol was determined by enzymatic method (Abbott Diagnostics) and HDLcholesterol by accelerator selective detergent method (Abbott Diagnostics). Triglyceride concentration was measured by the glycerol phosphatase oxidase method (Abbott Diagnostics).

Serum insulin was determined by a solid-phase, two-site chemiluminescent immunometric assay (Siemens Medical Solutions Diagnostics, Milan, Italy).

Serum Ox-LDL levels were measured by a commercial enzyme-linked immunosorbent assay (Ox-LDL ELISA, Mercodia, Uppsala, Sweden) on a Sunrise ${ }^{\circledast}$ absorbance microplate reader (Tecan, Switzerland), according to the manufacturer's instructions [22].

Briefly, the assay is based on the sandwich technique in which two monoclonal antibodies are directed against separate antigenic determinants on the oxidized apolipoprotein B molecule. Each sample must first be diluted in sample buffer to a final dilution of $1 / 6561$. The Ox-LDL concentrations were then calculated based on a six-point standard curve (range 0-150.9 U/L).

Results were obtained by multiplying Ox-LDL concentrations with the dilution factor (e.g. 6.561) and expressed as unit per liter $(\mathrm{U} / \mathrm{L})$. As regard the performance characteristics of the assay, the detection limit was $0.6 \mathrm{U} / \mathrm{L}$ while recovery ranged between $85 \%$ and $107 \%$ (mean value 95\%). Mean intra- and inter-assay analytical imprecision was, respectively, $6.3 \%$ and $4.7 \%$ coefficient of variation (CV). 
Abnormalities in lipid fasting levels were considered for TG values exceeding the 95th percentile and HDL cholesterol values below the 5th percentile for age and sex [23]. Impaired insulin sensitivity (ISI) was defined with HOMA-IR that exceeded the 97.5th percentile for age and sex [24].

\section{MetS definition}

MetS was diagnosed according to Weiss using the criteria modified from those of the National Cholesterol Education Program's Adult Treatment Panel III (NCEP-ATPIII) and the WHO [25]. Patients were classified as having MetS if they met three or more of the following criteria for age and sex: $\mathrm{BMI}>97$ th percentile, triglyceride levels $>95$ th percentile, HDL cholesterol level $<5$ th percentile, SBP and/or DBP $>95$ th percentile and impaired glucose tolerance.

In this recent definition by Weiss et al. [25] BMI was chosen as a criterion for the MetS because it correlates with visceral lipid distribution, blood pressure and dyslipidemia. Although waist circumference is a good predictor of visceral adiposity in children, it might not detect differences in body proportions related to puberty and therefore no normative values exist for children and adolescents. As marker of glucometabolic derangement, a pathological level of fasting blood glucose (FBG) and/or alteration of ISI were selected because impaired fasting glucose is rare in childhood and insulin resistance precedes glucose abnormalities and plays an important role in the pathogenesis of the transition from normal glucose tolerance to impaired glucose tolerance [26]. The euglycemic-hyperinsulinemic clamp is the gold standard for measuring insulin resistance, but this study is invasive, time consuming and difficult to apply to children. For these reasons, we utilized HOMA-IR as surrogate marker of insulin resistance/ sensitivity $[3,25]$.

Finally, blood pressure and fasting lipid levels were compared with population norms adjusted for age and sex.

This study was conducted according to the Good Clinical Practice guidelines and was approved by the Human Ethic Committees of Fondazione IRCCS Policlinico S. Matteo of Pavia (Protocol number: 20150005231). The informed written consent of a parent or legal guardian was required for subjects aged $<18$, and the subjects aged $\geq 8$ were asked to give their written assent. Patients' parents and patients $>8$ years gave their written consent to data publication. Our Ethics Committee also approved data publication.

\section{Statistical analysis}

Data quality control and statistical analyses were performed using STATA 11 (StataCorp, College Station, TX, USA). Descriptive statistics representing means, standard deviations, percentages and ranges were computed.

The analysis of variance (ANOVA), Student's t-test and the Kruskal-Wallis (in case of non-normality of the distributions tests) were used to verify that patients' characteristics did not differ significantly between the groups, taking into account the effect of sex and age, as possible confounders. The $\chi^{2}$-test was used to test the statistical significant differences of altered percentage values among the groups.
A multiple regression analysis was applied to evaluate the association between serum Ox-LDL levels and auxological/biochemical parameters, taking into account the effect of age and sex as possible confounders.

\section{Results}

\section{Auxological and metabolic parameters}

Based on the BMI percentiles threshold, 49 of the 178 patients $(27.5 \%)$ were normal weight, $76(42.7 \%)$ were overweight and 53 (29.8\%) were obese.

Auxological and metabolic parameters of the three groups are reported in Table 1 . Subjects were well matched for age and sex.

Concerning oxidative damage, children with obesity showed higher Ox-LDL levels compared to normal weight and overweight subjects $(\mathrm{p}<0.05)$. However, serum Ox-LDL levels were not significantly correlated with sex, age and pubertal status.

Regression analysis showed a significant correlation between Ox-LDL levels, BMI $(p=0.03, r=0.15)$, WC $(p=0.01, r=0.18)$ and W/HtR $(p=0.001, r=0.22)$; however, multiple regression analysis identified $\mathrm{W} /$ HtR (CI 95\% 13.3-151.5, p=0.02) as the only auxological independent factor for increased serum Ox-LDL levels (Figure 1).

Concerning routine biochemical parameters, a significant correlation $(\mathrm{p}<0.001)$ was noted between Ox-LDL and TG $(r=0.44)$, total cholesterol $(r=0.63)$ and LDL-cholesterol $(r=0.68)$. The exploratory multivariable regression analysis identified TG (CI 95\%: 0.04-0.15, $\mathrm{p}=0.001$ ) and LDL-cholesterol (CI 95\%: 0.19-0.54, p<0.001) as biochemical independent predictors of increased plasma Ox-LDL levels. In addition, on an average increase of $1 \mathrm{mg} /$ $\mathrm{dL}$ of LDL-cholesterol, Ox-LDL increases by $0.40 \mathrm{U} / \mathrm{L}$, while on an average increase of $1 \mathrm{mg} / \mathrm{dL}$ of TG, Ox-LDL increases by $0.10 \mathrm{U} / \mathrm{L}$.

No correlation between serum Ox-LDL levels and blood pressure was detected.

\section{Metabolic syndrome}

In our study population, the overall prevalence of MetS was $15.2 \%(n=27)$; particularly, MetS was present in 9.2\% $(\mathrm{n}=9)$ and in $35.2 \%(\mathrm{n}=18)$ of subjects with overweight and obesity, respectively $(\mathrm{p}=0.001)$. No normal weight subjects met the criteria for MetS. 
Table 1: Auxological and biochemical parameters in our study population.

\begin{tabular}{|c|c|c|c|}
\hline Parameters & $\begin{array}{r}\text { Normal weight } \\
(\mathrm{n}=49)\end{array}$ & $\begin{array}{r}\text { Overweight } \\
(n=76)\end{array}$ & $\begin{array}{l}\text { Obesity } \\
(n=53)\end{array}$ \\
\hline Age, years & $11.2 \pm 2.5$ & $12.2 \pm 2.5$ & $11.5 \pm 2.3$ \\
\hline Gender, M/F & $27 / 22$ & $35 / 41$ & $28 / 25$ \\
\hline \multicolumn{4}{|l|}{ Pubertal stages, $\mathrm{M} / \mathrm{F}$} \\
\hline Tanner I & $11 \mathrm{M} / 2 \mathrm{~F}$ & $4 \mathrm{M} / 2 \mathrm{~F}$ & $7 \mathrm{M} / 4 \mathrm{H}$ \\
\hline Tanner II-III & $12 \mathrm{M} / 14 \mathrm{~F}$ & $19 \mathrm{M} / 15 \mathrm{~F}$ & $16 \mathrm{M} / 10$ \\
\hline Tanner IV-V & $4 \mathrm{M} / 6 \mathrm{~F}$ & $12 \mathrm{M} / 24 \mathrm{~F}$ & $5 \mathrm{M} / 11$ \\
\hline \multirow[t]{2}{*}{$W C, \mathrm{~cm}^{\mathrm{a}, \mathrm{b}}$} & $62.7 \pm 7.5$ & $79.2 \pm 7.7$ & $92.9 \pm 16$ \\
\hline & {$[2.0]$} & {$[73.7]$} & {$[100$} \\
\hline \multirow[t]{2}{*}{$\mathrm{W} / \mathrm{HtR}^{\mathrm{a}, \mathrm{b}}$} & $0.4 \pm 0$ & $0.5 \pm 0$ & $0.6 \pm 0.1$ \\
\hline & {$[0]$} & [57.9] & {$[100$} \\
\hline \multirow[t]{2}{*}{ Fasting glucose, $\mathrm{mg} / \mathrm{dL}^{\mathrm{b}}$} & $74 \pm 7.4$ & $75.7 \pm 9$ & $75.6 \pm 10.5$ \\
\hline & {$[0]$} & [3.9] & {$[18.9$} \\
\hline \multirow[t]{2}{*}{ Insulin, $\mu \mathrm{U} / \mathrm{L}^{\mathrm{a}, \mathrm{b}}$} & $5.9 \pm 3.4$ & $11.8 \pm 11.9$ & $15.9 \pm 9.6$ \\
\hline & {$[0]$} & [13.2] & {$[49.1]$} \\
\hline \multirow[t]{2}{*}{ HOMA-IR } & $1.1 \pm 0.7$ & $2.2 \pm 2.1$ & $3 \pm 2$ \\
\hline & {$[0]$} & {$[10.5]$} & {$[37.7$} \\
\hline \multirow[t]{2}{*}{ Triglycerides, mg/dL ${ }^{\mathrm{a}, \mathrm{b}}$} & $49.4 \pm 17.2$ & $65.1 \pm 27$ & $86.5 \pm 45$ \\
\hline & {$[4.0]$} & {$[14.5]$} & {$[20.8$} \\
\hline \multirow[t]{2}{*}{ HDL-cholesterol, mg/dLa,b } & $56 \pm 10.3$ & $50.7 \pm 11.3$ & $45.5 \pm 9.4$ \\
\hline & {$[4.0]$} & {$[17.1]$} & {$[39.6$} \\
\hline \multirow[t]{2}{*}{ LDL-cholesterol, $\mathrm{mg} / \mathrm{dL}^{\mathrm{a}}$} & $88.9 \pm 23.4$ & $93.5 \pm 27$ & $102.1 \pm 20.2$ \\
\hline & {$[0]$} & {$[2.6]$} & {$[0$} \\
\hline \multirow[t]{2}{*}{ Total cholesterol, mg/dL } & $157.6 \pm 28.3$ & $158.3 \pm 29.6$ & $163.3 \pm 25.8$ \\
\hline & {$[0]$} & {$[2.6]$} & {$[0$} \\
\hline$O x-L D L, U / L^{a}$ & $44.4 \pm 11.6$ & $50.6 \pm 18.9$ & $52.8 \pm 15.9$ \\
\hline \multirow[t]{2}{*}{ Systolic pressure, $\mathrm{mmHg}^{\mathrm{a}}$} & $101.9 \pm 9.1$ & $110 \pm 12.9$ & $112.9 \pm 10.6$ \\
\hline & [4] & {$[6.6]$} & {$[3.8$} \\
\hline \multirow[t]{2}{*}{ Diastolic pressure, $\mathrm{mmHg}^{\mathrm{a}}$} & $63.6 \pm 8.9$ & $67.9 \pm 7.3$ & $71.3 \pm 8.4$ \\
\hline & {$[8.2]$} & {$[10.5]$} & {$[22.6$} \\
\hline
\end{tabular}

Data are reported as mean \pm standard deviation. In squared brackets are reported the percentages of altered values (under or above the relevant percentile curves for age and sex, as described in Patients and methods section). ${ }^{a}$ p-Value $<0.05$ : age and sex adjusted analysis of variance (ANOVA). ${ }^{\mathrm{b}} \mathrm{p}$-Value $<0.05: \chi^{2}$-test analysis. WC, waist circumference; Ox-LDL, oxidized LDL cholesterol; W/HtR, waist-to-height ratio. Subjects were divided on the basis of the threshold BMI [15].

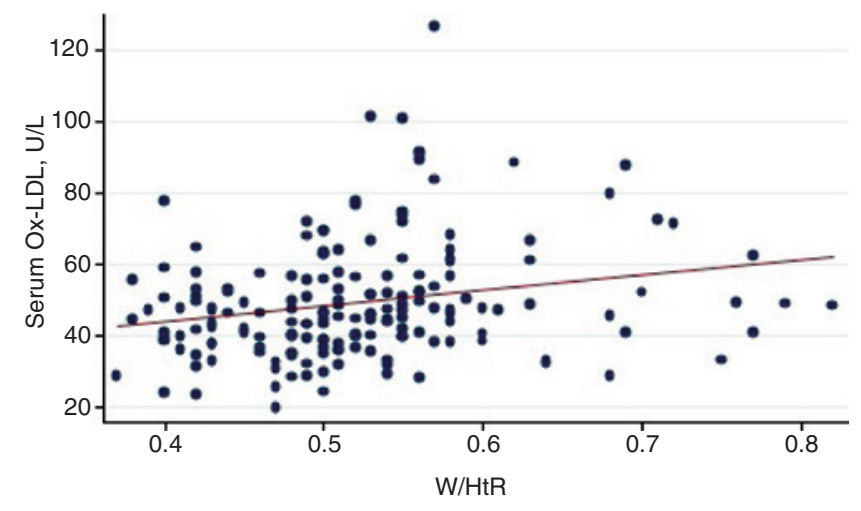

Figure 1: Multiple regression analysis.

Serum Ox-LDL levels are the dependent variable while the following variables are included as independent factors: $B M I\left(\mathrm{~kg} / \mathrm{m}^{2}\right)$, WC $(\mathrm{cm})$ and W/HtR. Only W/HtR is an independent factor for increased Ox-LDL levels (CI 95\% 13.3-151.5, $\mathrm{p}=0.02)$.
Auxological and metabolic parameters in patients with and without MetS are reported in Table 2. Subjects were well matched for age and sex.

Two subjects $(1 \mathrm{M} / 1 \mathrm{~F})$ in Tanner stage I and 17 subjects (10 M/7 F) in Tanner stages II and III had already developed MetS.

Concerning oxidative damage, serum Ox-LDL levels were higher in subjects with MetS than in those without MetS approaching, but not reaching significance $(\mathrm{p}=0.06)$.

\section{Discussion}

MetS is emerging as a new clinical problem within pediatric practice. In this report, we confirm an increased 
Table 2: Auxological and biochemical parameters in our study population.

\begin{tabular}{|c|c|c|}
\hline Parameters & $\begin{array}{r}\text { Without metabolic } \\
\text { syndrome } \\
(n=151)\end{array}$ & $\begin{array}{r}\text { With metabolic } \\
\text { syndrome } \\
(n=27)\end{array}$ \\
\hline Age, years & $11.6(2.4)$ & $12.4(2.6)$ \\
\hline Gender, M/F & $74 / 77$ & $16 / 11$ \\
\hline Waist circumference, $\mathrm{cm}^{\mathrm{a}, \mathrm{b}}$ & $\begin{array}{r}76.2(14.1) \\
{[57.6]}\end{array}$ & $\begin{array}{r}93.2(16.8) \\
{[85.2]}\end{array}$ \\
\hline Waist-to-height ratio ${ }^{\mathrm{a}, \mathrm{b}}$ & $\begin{array}{r}0.5(0.1) \\
{[49.0]}\end{array}$ & $\begin{array}{r}0.6(0.1) \\
{[85.2]}\end{array}$ \\
\hline \multicolumn{3}{|l|}{ Pubertal stages } \\
\hline Tanner I & $21 \mathrm{M} / 7 \mathrm{~F}$ & $1 \mathrm{M} / 1 \mathrm{~F}$ \\
\hline Tanner II-III & $37 \mathrm{M} / 32 \mathrm{~F}$ & $10 \mathrm{M} / 7 \mathrm{~F}$ \\
\hline Tanner IV-V & $16 \mathrm{M} / 38 \mathrm{~F}$ & $5 \mathrm{M} / 3 \mathrm{~F}$ \\
\hline Fasting glucose, $\mathrm{mg} / \mathrm{dL}^{\mathrm{a}, \mathrm{b}}$ & $\begin{array}{r}74.4(7.7) \\
{[0]}\end{array}$ & $\begin{array}{r}79.6(14) \\
{[14.8]}\end{array}$ \\
\hline Insulin, $\mu \mathrm{U} / \mathrm{L}^{\mathrm{a}, \mathrm{b}}$ & $\begin{array}{r}9.5(5.4) \\
{[12.6]}\end{array}$ & $\begin{array}{r}23(19.7) \\
{[85.2]}\end{array}$ \\
\hline HOMA-IR & $\begin{array}{r}1.8(1) \\
{[7.2]}\end{array}$ & $\begin{array}{r}4.5(3.6) \\
{[66.7]}\end{array}$ \\
\hline Triglycerides, mg/dL $\mathrm{L}^{\mathrm{a}, \mathrm{b}}$ & $\begin{array}{r}61.5(25.1) \\
{[4.0]}\end{array}$ & $\begin{array}{r}102.3(54.5) \\
{[44.4]}\end{array}$ \\
\hline HDL-cholesterol, mg/dL ${ }^{\mathrm{a}, \mathrm{b}}$ & $\begin{array}{r}52.3(10.5) \\
{[11.0]}\end{array}$ & $\begin{array}{r}39.9(8.2) \\
{[74.1]}\end{array}$ \\
\hline LDL-cholesterol, mg/dL & $\begin{array}{r}95(24.8) \\
{[1.3]}\end{array}$ & $\begin{array}{r}95.7(23.7) \\
{[0]}\end{array}$ \\
\hline Total cholesterol, mg/dL & $\begin{array}{r}160.7(27.7) \\
{[1.3]}\end{array}$ & $\begin{array}{r}154.7(29.9) \\
{[0]}\end{array}$ \\
\hline Ox-LDL, U/L & $48.8(15.9)$ & $54.1(19.4)$ \\
\hline Systolic pressure, mmHga,b & $\begin{array}{r}106.7(10.6) \\
{[2.0]}\end{array}$ & $\begin{array}{r}120.9(13.1) \\
{[22.3]}\end{array}$ \\
\hline Diastolic pressure, $\mathrm{mmHg}^{\mathrm{a}, \mathrm{b}}$ & $\begin{array}{r}66.6(7.7) \\
{[7.3]}\end{array}$ & $\begin{array}{r}74.6(9.8) \\
{[48.1]}\end{array}$ \\
\hline
\end{tabular}

Data are reported as mean and (standard deviation). In squared brackets are reported the percentages of altered values (under or above the relevant percentile curves for age and sex, as described in Materials and Methods section). ${ }^{a} \mathrm{p}$-Value $<0.05$ : age and sex adjusted analysis of variance (ANOVA). ${ }^{\text {b}} p$-Value $<0.05: \chi^{2}$-test analysis. WC, waist circumference; Ox-LDL, oxidized LDL cholesterol.

prevalence of MetS in participants with obesity compared to normal weight children. Children with obesity showed higher Ox-LDL levels compared to normal weight and overweight subjects, with a limited relation between Ox-LDL and MetS. W/HtR, tryglicerides and LDL-cholesterol were independent predictors of increased plasma Ox-LDL levels.

The synergistic effects of the MetS components may lead to cardiovascular disease as children with obesity become young adults [5-7], and this may be announced by an increased OS biomarkers level [7, 8]. OS may play a causative role in the development of obesity and co-morbidities [9-12]. The possible contributors to OS in obesity include hyperglycemia, elevated lipid levels, chronic low grade inflammation with increased circulating cytokines produced by visceral adipose depots as well as myokines production by skeletal muscles, in those with low physical activity level and higher weigh to carry, endothelial dysfunction, unhealthy diets [7-12].

Evidence has demonstrated that OS levels are elevated in adults with obesity [12], but pediatric data in this area are limited. In our sample, Ox-LDL level was measured as a marker of oxidative damage and it was found to be elevated in children with obesity, particularly in those with visceral adiposity. Each element of the MetS worsens as waist circumference increases, independently of age, sex and pubertal status. Recent studies performed in adolescents with obesity showed that abdominal fat, rather than BMI, may be the main cause of metabolic impact [19, 27]. This adverse metabolic phenotype is associated to OS, severe insulin resistance and alterations in glucose and lipid metabolism [25]. The correlation between Ox-LDL levels and W/HtR confirm these data and suggest that Ox-LDL high values may reflect and predict MetS development in children too.

In adults, Ox-LDL is strongly associated whit MetS and its components independently of central obesity and insulin resistance and could be useful as early predictive marker of cardiometabolic abnormalities [12]. In our sample Ox-LDL levels were higher in subjects with MetS, despite the difference in children with and without MetS did not reach the usually accepted level of clinical significance. In pediatric ages MetS components were rarely all present in early stage and this may have influenced the limited level of clinical significance; moreover higher Ox-LDL values in patients with MetS as well as the association between Ox-LDL, tryglicerides and W/HtR, claim that increased oxidative damage and inflammation might be involved in the development of the MetS.

The association between Ox-LDL and TG, the strongest found among the MetS components, may be partly due to their common participation in lipid pathway, beyond TG participation in the MetS. Hypertriglyceridemia is associated with higher levels of small dense LDL, which are particularly prone to oxidation and have been proven more atherogenic than larger LDL particles. Moreover, the hypothesis that tryglicerides may be an earlier dismetabolic marker is not excluded [28]. Lemieux et al. [29] demonstrated that the combination of only two MetS components, increased TG levels and WC, defined as hypertriglyceridemic waist phenotype, were able to predict cardiovascular risk in adult men. Despite high tryglicerides levels being a modifiable cardiovascular risk factor, it is undeniable that they contribute to oxidative damage. 
The free fatty acids available in situations leading to MetS, induce insulin resistance, inhibiting insulin-mediated glucose uptake, that is associated with other metabolic variables [25].

In addition to the impact of lipid profiles, OS has also been linked with important cardiovascular risk factors [8$12,30]$. Vascular dysfunction is documented as an important process in driving the pathologies associated with obesity and hypertension. Human and animal models of obesity have demonstrated altered vascular contractility and decreased dilator function promoting hypertension. The mechanisms causing this vascular dysfunction involve the combinatorial effects of OS and inflammatory signaling, widely recognized as major players in the pathogenesis of cardiovascular diseases and overt hypertension [31]. In our children with obesity, no correlation between serum Ox-LDL levels and blood pressure was detected, providing minimal evidence of such association with early, pre-clinical abnormalities of blood pressure vs. overt hypertension.

We recognize that there are some study limitations starting from the sample, relatively small, which did not allows to us to build cut-off values or reference intervals for Ox-LDL levels in our study population. Therefore, expanding sampling is mandatory.

Additionally, we particularly want to underline that we did not evaluate OS status but oxidative damage. In fact, as previously reported in the literature [32], under normal physiological conditions, reactive oxygen species (ROS) can be eliminated or inactivated in vivo by different antioxidant systems. However, OS occurs either when there is an increase of ROS or a decrease in the antioxidant systems or damage induced by OS, such as Ox-LDL production [33], as a consequence of oxidants-antioxidant imbalance [32]. Based on these considerations, the authors think that is more correct to refert to an oxidative damage rather than OS, underlying that further studies, in order to evaluate the antioxidant systems are mandatory.

However, given the few data so far reported in pediatric ages, we believe that the observed increased levels of Ox-LDL, approaching conventional levels of significance, in subjects with MetS in our population, offer a good starting point for further investigation on oxidative damage and the development of chronic degenerative diseases in the young ones. Changes in oxidative damage biomarkers, such as Ox-LDL, in pediatric ages, could have a potential clinical meaning and may be an early marker of long-term chronic diseases, like MetS, and cardiometabolic risk in those with an adverse metabolic phenotype.

Targeting actions toward OS biomarkers, linked to insulin-related metabolic processes, could lead to more homogeneous evidence to tackle obesity and overweight in children, confirming the need of a prospective change of the obesity concept already suggested by other authors [34] for efficient global action.

Author contributions: All the authors have accepted responsibility for the entire content of this submitted manuscript and approved submission.

Research funding: None declared.

Employment or leadership: None declared.

Honorarium: None declared.

Competing interests: The funding organization(s) played no role in the study design; in the collection, analysis, and interpretation of data; in the writing of the report; or in the decision to submit the report for publication.

\section{References}

1. Mameli C, Zuccotti GV, Carnovale C, Galli E, Nannini P, et al. An update on the assessment and management of metabolic syndrome, a growing medical emergency in paediatric populations. Pharmacol Res 2017;119:99-117.

2. Titmuss AT, Srinivasan S. Metabolic syndrome in children and adolescents: old concepts in a young population. J Paediatr Child Health 2016;52:928-34.

3. Calcaterra V, Klersy C, Muratori T, Telli S, Caramagna C, et al. Prevalence of metabolic syndrome (MS) in children and adolescents with varying degrees of obesity. Clin Endocrinol 2008;68:868-72.

4. Yeste D, Carrascosa A. Obesity-related metabolic disorders in childhood and adolescence. An Pediatr (Barc) 2011;75:135.e1-9.

5. Rutigliano I, Vinci R, De Filippo G, Mancini M, Stoppino L, et al. Metabolic syndrome, hepatic steatosis, and cardiovascular risk in children. Nutrition 2017;36:1-7.

6. Agirbasli M, Tanrikulu AM, Berenson GS. Metabolic syndrome: bridging the gap from childhood to adulthood. Cardiovasc Ther 2016;34:30-6.

7. Higgins V, Adeli K. Pediatric metabolic syndrome: pathophysiology and laboratory assessment. Electron J Int Fed Clin Chem 2017;28:25-42.

8. Ho E, Karimi Galougahi K, Liu CC, Bhindi R, Figtree GA. Biological markers of oxidative stress: applications to cardiovascular research and practice. Redox Biol 2013;1:483-91.

9. Yadav UC, Rani V, Deep G, Singh RK, Palle K. Oxidative stress in metabolic disorders: pathogenesis, prevention, and therapeutics. Oxid Med Cell Longev 2016;2016:9137629.

10. Jadeja RN, Devkar RV, Nammi S. Oxidative stress in liver diseases: pathogenesis, prevention, and therapeutics. Oxid Med Cell Longev 2017;2017:8341286.

11. Holvoet P, De Keyzer D, Jacobs DR Jr. Oxidized LDL and the metabolic syndrome. Future Lipidol 2008;3:637-49.

12. Hurtado-Roca Y, Bueno H, Fernandez-Ortiz A, Ordovas JM, Ibañez $B$, et al. Oxidized LDL is associated with metabolic syndrome traits independently of central obesity and insulin resistance. Diabetes 2017;66:474-82. 
13. EFSA Panel on dietetic Products, Nutrition and Allergies (NDA). Guidance on the scientific requirements for health claims related to antioxidants, oxidative damage and cardiovascular health. EFSA J 2011;9:2474.

14. Kelishadi R, Hashemipour M, Adeli K, Tavakoli N, MovahedianAttar A, et al. Effect of zinc supplementation on markers of insulin resistance, oxidative stress, and inflammation among prepubescent children with metabolic syndrome. Metab Syndr Relat Disord 2010;8:505-10.

15. Cole TJ, Lobstein T. Extended international (IOFT) body mass index cut-offs for thinness, overweight and obesity. Pediatric Obes 2012;7:284-94.

16. Valerio G, Balsamo A, Baroni MG, Brufani C, Forziato C, et al. Childhood obesity classification systems and cardiometabolic risk factors: a comparison of the Italian, World Health Organization and International Obesity Task Force references. Ital J Pediatr 2017;43:19.

17. Marshall WA, Tanner JM. Variations in patterns of pubertal changes in boys. Arch Dis Child 1969;45:13-23.

18. Marshall WA, Tanner JM. Variations in patterns of pubertal changes in girls. Arch Dis Child 1969;44:291-303.

19. Maffeis C, Banzato C, Talamini G, Obesity Study Group of the Italian Society of Pediatric Endocrinology and Diabetology. Waist-to-height ratio, a useful index to identify high metabolic risk in overweight children. J Pediatr 2008;152:207-13.

20. National High Blood Pressure Education Program Working Group on High Blood Pressure in Children and Adolescents. The fourth report on the diagnosis, evaluation, and treatment of high blood pressure in children and adolescents. Pediatrics 2004;114:555-76.

21. Matthews DR, Hosker JP, Rudenski AS, Naylor BA, Treacher DF, et al. Homeostasis model assessment: insulin resistance and -cell function from fasting plasma glucose and insulin concentrations in man. Diabetologia 1985;28:412-9.

22. Holvoet P, Macy E, Landeloos M, Jones D, Jenny NS, et al. Analytical performance and diagnostic accuracy of immunometric assays for the measurement of circulating oxidized LDL. Clin Chem 2006;52:760-4.

23. Expert Panel on Detection, Evaluation, and Treatment of High Blood Cholesterol in Adults (Adult Treatment Panel III) final report. National Cholesterol Education Program (NCEP) Expert Panel on Detection, Evaluation, and Treatment of High Blood Cholesterol in Adults (Adult Treatment Panel III). Third Report of the National Cholesterol Education Program (NCEP). Circulation 2002;106:3143-421.

24. d'Annunzio G, Vanelli M, Pistorio A, Minuto N, Bergamino L, et al. Insulin resistance and secretion indexes in healthy Italian children and adolescents: a multicentre study. Acta Biomedica 2009;80:21-8.

25. Weiss R, Dziura J, Burgert TS, Tamboriane WV, Taksali SE, et al. Obesity and the metabolic syndrome in children and adolescents. N Engl J Med 2004;350;23.

26. The Expert Committee on the Diagnosis and Classification of Diabetes Mellitus. Report of the expert committee on the diagnosis and classification of diabetes mellitus. Diabetes Care 1999;22(Suppl 1):S5-19.

27. Teixeira FC, Pereira FE, Pereira AF, Ribeiro BG. Metabolic syndrome's risk factors and its association with nutritional status in schoolchildren. Prev Med Rep 2017;6:27-32.

28. Hübers M, Geisler C, Plachta-Danielzik S, Müller MJ. Association between individual fat depots and cardio-metabolic traits in normal- and overweight children, adolescents and adults. Nutr Diabetes 2017;7:e267.

29. Lemieux I, Poirier P, Bergeron J, Alméras N, Lamarche $B$, et al. Hypertriglyceridemic waist: a useful screening phenotype in preventive cardiology? Can J Cardiol 2007;23(Suppl B):23B-31B.

30. Ceriello A. Possible role of oxidative stress in the pathogenesis of hypertension. Diabetes Care 2008;31(Suppl 2):S181-4.

31. Reho JJ, Rahmouni K. Oxidative and inflammatory signals in obesity-associated vascular abnormalities. Clin Sci (Lond) 2017;131:1689-700.

32. Fernández-García JC, Cardona F, Tinahones FJ. Inflammation, oxidative stress and metabolic syndrome: dietary modulation. Curr Vasc Pharmacol 2013;11:906-19.

33. EFSA Panel on dietetic Products, Nutrition and Allergies (NDA). EFSA J 2011;9:2474.

34. Camancho S, Ruppel A. Is the calorie concept a real solution to the obesity epidemic? Glob Health Action 2017;10:1289650. 